\title{
Contributions to study the dimensional precision of the thin shafts obtained by fused deposition modelling
}

\author{
Daniel - Constantin ANGHEL , Gina Mihaela SICOE \\ University of Pitesti, Pitesti, Romania \\ ${ }^{*}$ Corresponding author e-mail: daniel.anghel@upit.ro \\ Article history \\ Received 15.06.2019 \\ Accepted 20.08.2019 \\ DOI https://doi.org/10.26825/bup.ar.2019.002
}

\begin{abstract}
This article presents a study on the dimensional accuracy of the thin shafts obtained by the Fused Deposition Modelling process. The study is based on the execution of a number of 27 specimens with various values of the parameters: layer thickness, H/D ratio, infill density. These combinations of values were established according to a Taguchi plan.

The specimens were modeled in CatiaV5, then the Z-Suite software generated the codes for printing by the Zortrax M200 3D printer. Z-ABS was used as the material. After testing, these were post-processed and measured.

The data were analyzed with the MiniTab software and with the Artificial Neural Networks Toolbox from MATLAB.
\end{abstract}

Keywords: Fused Deposition Modelling, Artificial Neural Networks, 3D Printing

\section{Introduction}

Additive Manufacturing is a new technology, opposed to Subtractive Manufacturing and allows the production of parts with complex shapes and structures.

The filament material passes through an extrusion head and is heated to near its melting point. This material is then removed through the end of the head and stored on the machine table in the form of a single material wire; these "threads" are deposited one after another to create the layer. Once the layer is completed, the construction table descends by one layer and the process continues until the next layer is completed [MOH 16]. Parts with downward facing surfaces require substantial support. While in other processes these supports are automatically generated, in the case of FDM material other than the part is used. The material is a plastic (ABS, PLA etc), and the parts built during the process have a resistance of $80 \%$ of that of the original material. Other materials include wax, medical ABS and an elastomer [KRI 06], [KAR 14], [KHA 05] and [TSO 16].

Advantages: good accuracy, functional materials, medium range of materials, easy maintenance, topological optimization, multi-material parts, customized table, reinforced part.

Disadvantages: support material. 
The FDM technology is very complex, based on thermomechanical processes, on high speed and precise displacements [KUN 14]. This complexity causes FDM technology to have a number of problems that are still unclear, like: clogged extruder, layer shifting, weak infill, grinding filament, material stringing, overheating, inconsistent extrusion and so on.

In ours experiments we found some problems of dimensional accuracy on the parts realized by FDM. In order to determine the dimensional accuracy of the thin shafts obtained by FDM process, an experiment was made at University of Pitești, in the laboratory of Product design and development.

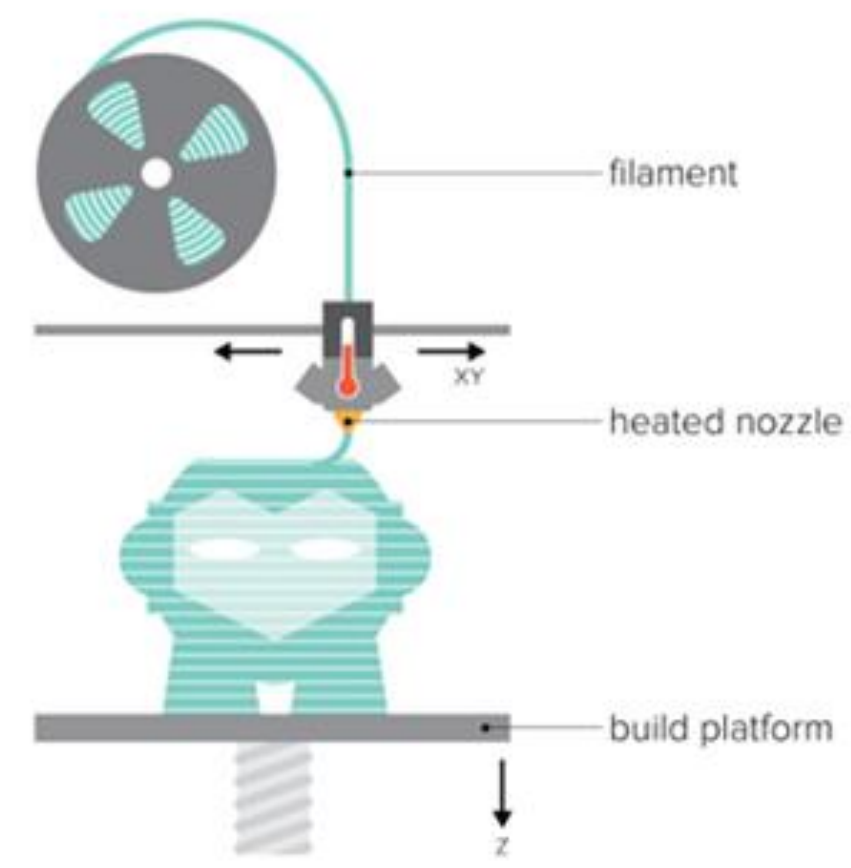

Figure 1. 3D Printing process

\section{The specimens used in the study}

The drawing of the specimens is presented in Figure 2.
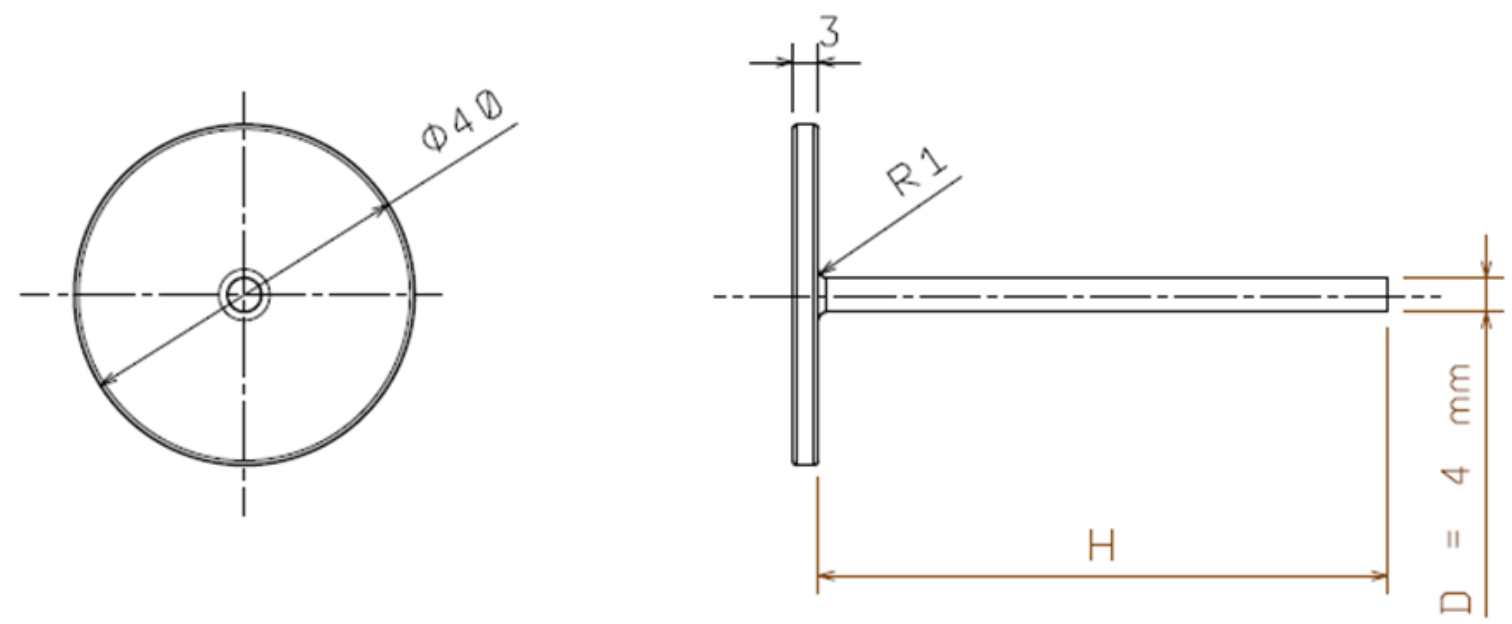

Figure 2. Drawing of the specimens

The 3D specimen is realized with Catia V5 software and after then the 3D model is imported in ZSuite software in order to generate the code for the 3D Printer ZORTRAX M200, figure 3. 


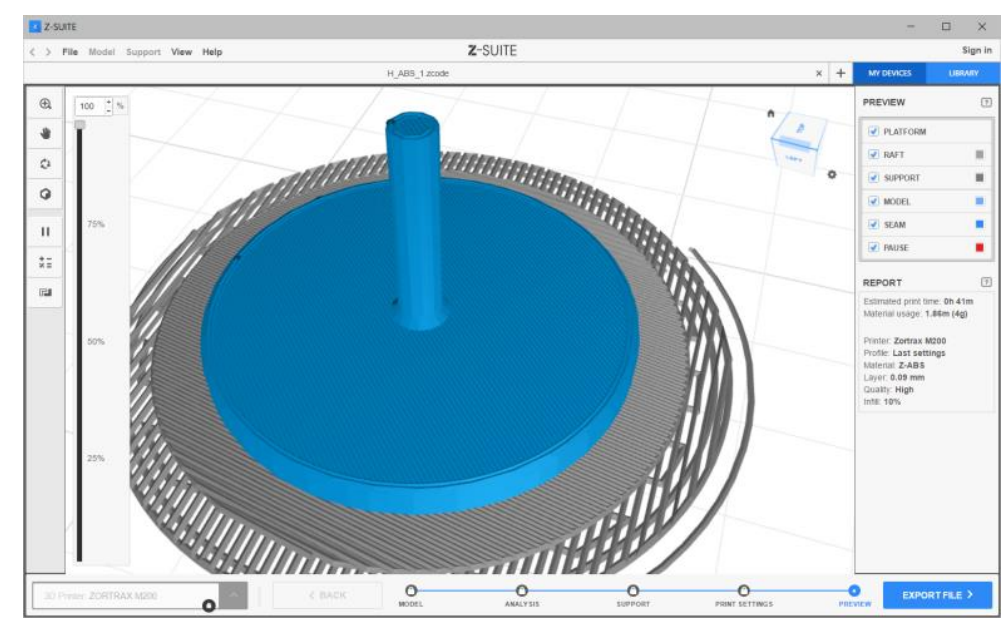

Figure 3. Z-Suite software

\section{Analysis and results}

\subsection{Planning and data analysis with Minitab}

With Minitab software we created a Taguchi plan for experimentation. This plan has 3 factors and for each factor exist 3 levels. Based on Taguchi plan, a number of 27 specimens was realized. The following parameters were chosen as inputs: layer thickness, H/D ratio, infill density and the output was the diameter measured on the top of the shaft, table 1 .

Table 1. Dataset for the experiment

\begin{tabular}{|c|c|c|c|c|}
\hline Exp. no. & $\begin{array}{l}\text { Layer thickness } \\
(\mathrm{mm})\end{array}$ & H/D Ratio & Infill density (\%) & Diameter $(\mathrm{mm})$ \\
\hline 1. & 0,09 & 5 & 10 & 4,09 \\
\hline 2. & 0,09 & 5 & 10 & 4,08 \\
\hline 3. & 0,09 & 5 & 10 & 4,08 \\
\hline 4. & 0,09 & 10 & 50 & 4,10 \\
\hline 5. & 0,09 & 10 & 50 & 4,11 \\
\hline 6. & 0,09 & 10 & 50 & 4,11 \\
\hline 7. & 0,09 & 15 & 90 & 4,15 \\
\hline 8. & 0,09 & 15 & 90 & 4,16 \\
\hline 9. & 0,09 & 15 & 90 & 4,16 \\
\hline 10. & 0,19 & 5 & 50 & 4,11 \\
\hline 11. & 0,19 & 5 & 50 & 4,12 \\
\hline 12. & 0,19 & 5 & 50 & 4,13 \\
\hline 13. & 0,19 & 10 & 90 & 4,14 \\
\hline 14. & 0,19 & 10 & 90 & 4,14 \\
\hline 15. & 0,19 & 10 & 90 & 4,13 \\
\hline 16. & 0,19 & 15 & 10 & 4,19 \\
\hline 17. & 0,19 & 15 & 10 & 4,21 \\
\hline 18. & 0,19 & 15 & 10 & 4,21 \\
\hline 19. & 0,14 & 5 & 90 & 4,09 \\
\hline 20. & 0,14 & 5 & 90 & 4,09 \\
\hline 21. & 0,14 & 5 & 90 & 4,11 \\
\hline 22. & 0,14 & 10 & 10 & 4,14 \\
\hline 23. & 0,14 & 10 & 10 & 4,13 \\
\hline 24. & 0,14 & 10 & 10 & 4,14 \\
\hline 25. & 0,14 & 15 & 50 & 4,18 \\
\hline 26. & 0,14 & 15 & 50 & 4,17 \\
\hline 27. & 0,14 & 15 & 50 & 4,18 \\
\hline
\end{tabular}


A regression analysis was realized: Diameter versus Layer height; Rapport H/D, Figure 4.

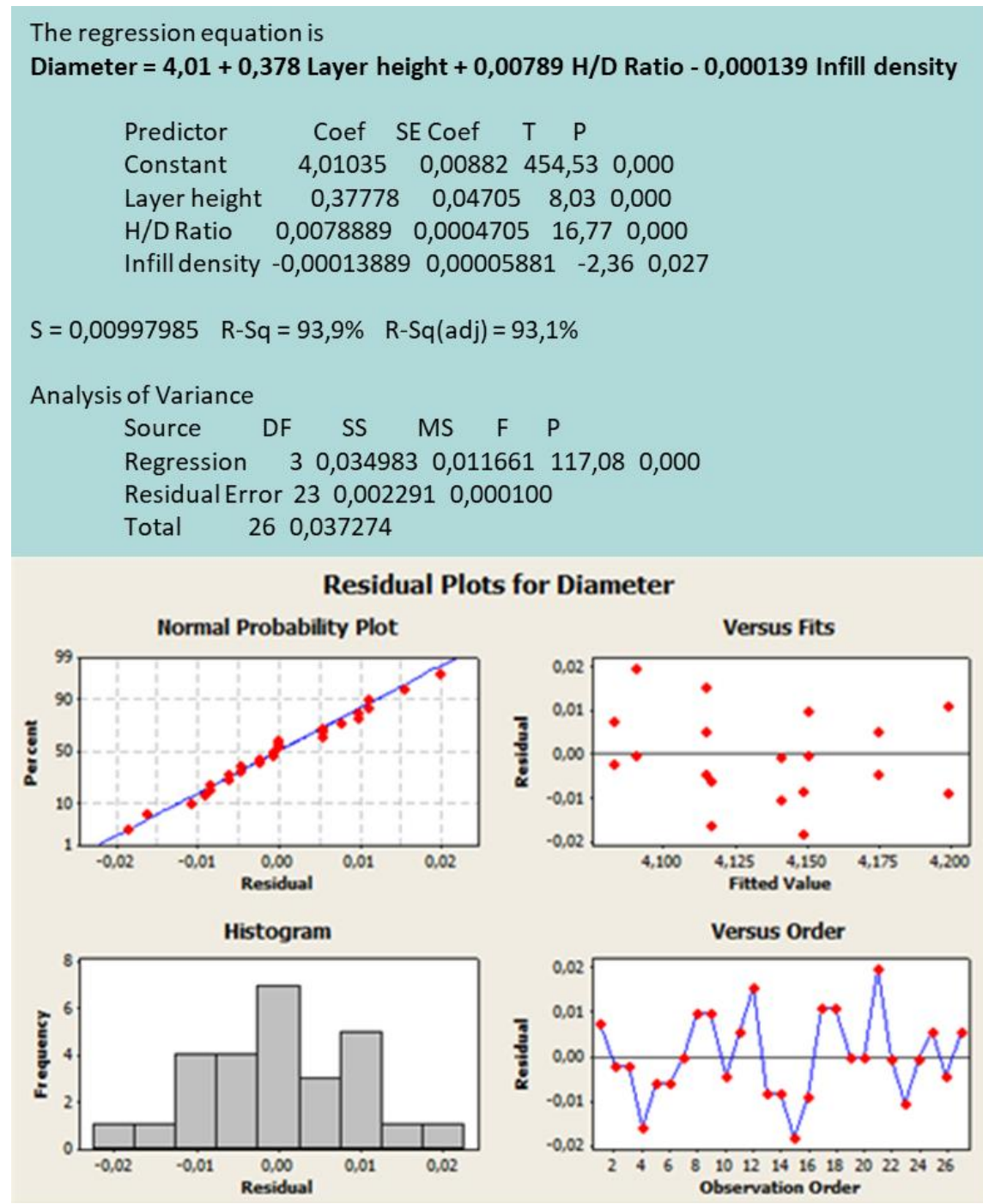

Figure 4. Rapport of regression analysis generated by Minitab software

\subsection{Data analysis with Artificial Neural Network Toolbox in MATLAB}

The data was imported into the MATLAB Artificial Neural Network Toolbox.

For the inputs of the network we created a matrix of 3x27 (Layer height, H/D Ratio and Infill density), and for the output of the network a matrix (vector) of 1x27 (Diameter) were created.

In order to train, test and validate the network, we divided the 27 data sets into 3 categories: $70 \%$ for training, $15 \%$ for testing and $15 \%$ for validation.

The Neural Network Fitting Tool was chosen because this tool has the possibility to randomly distribute the data sets in the three categories.

The network was trained using the "Bayesian-Regularization" backpropagation algorithm (ANG 19), figure 6 . This process is an iterative one, in this case, to train the network a number of 614 iterations was required. 


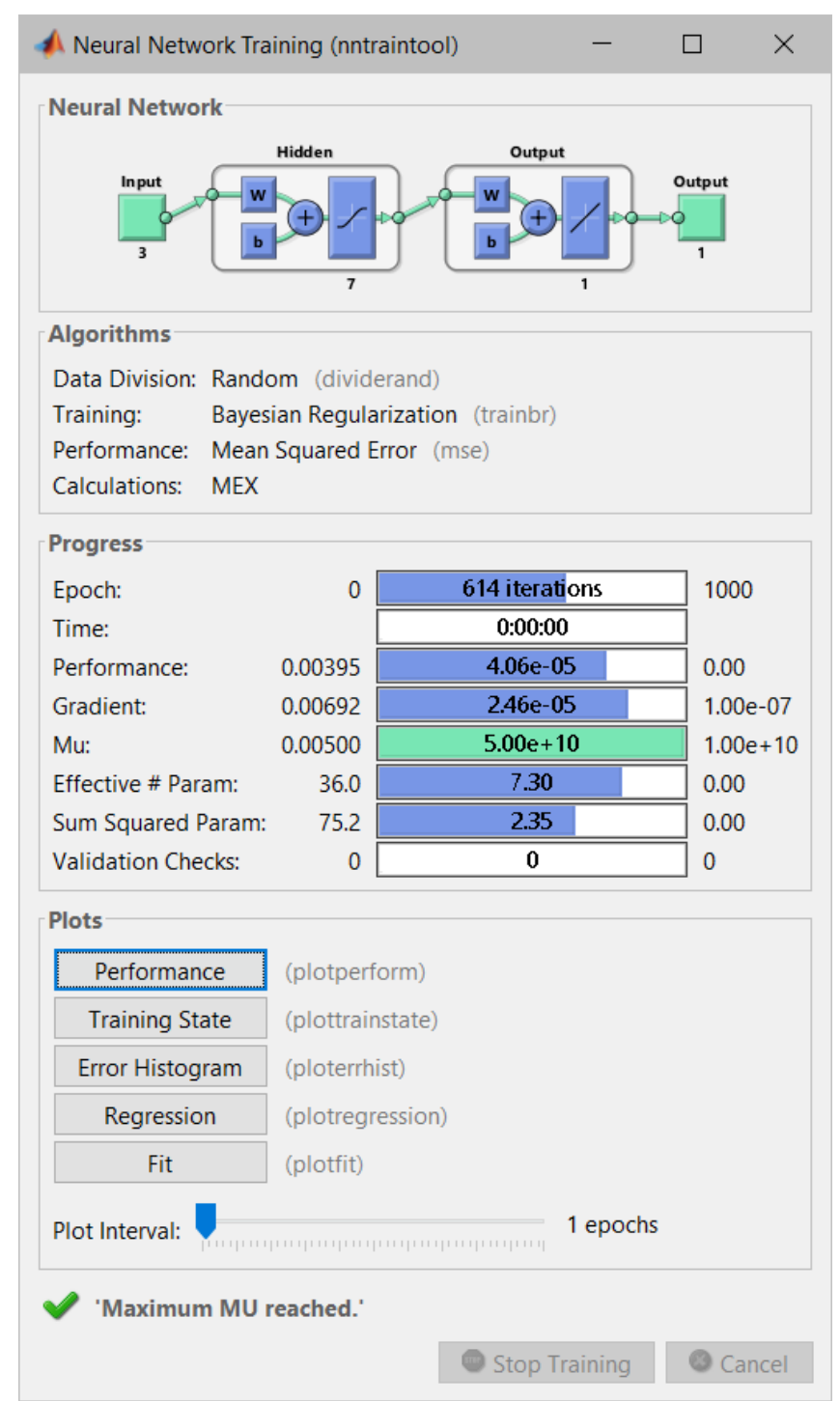

Figure 6. Training the neural network

When the training process is considered completed, the results are displayed in the Train Network window. The values for $\mathrm{R}$ and the mean square error are displayed.

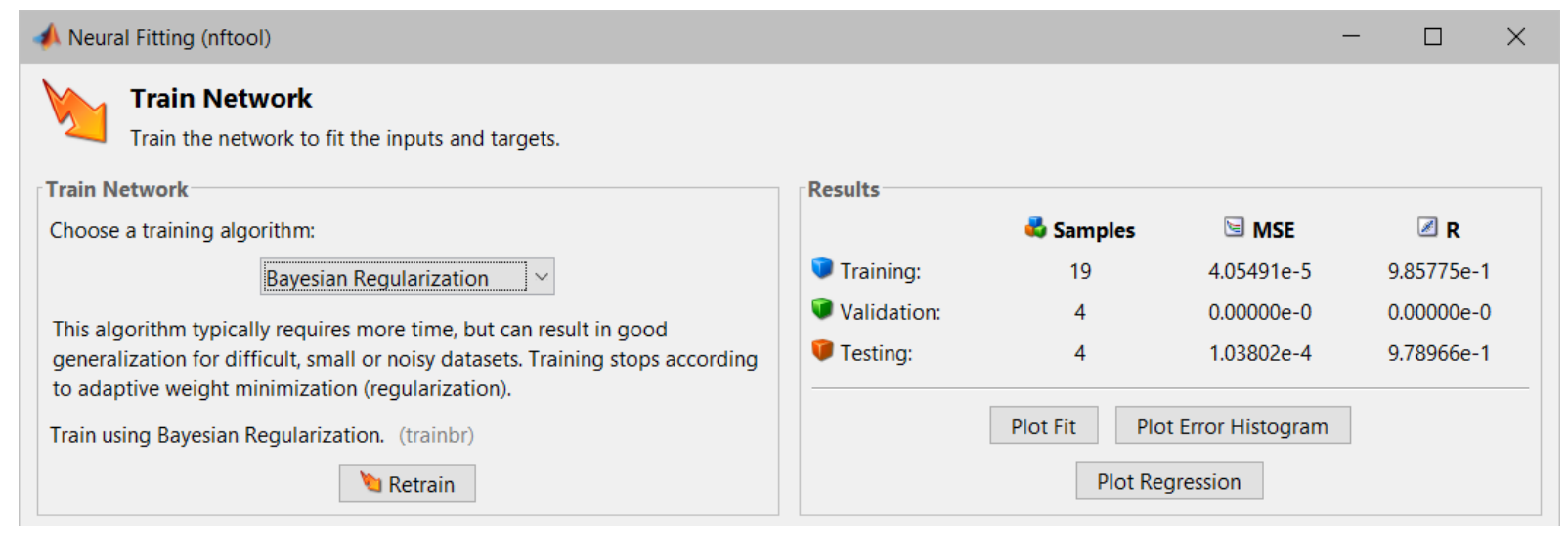

Figure 7. The results of network training, testing and validation

After the training, the network can be used by the designer "to ask" for advice in order to "simulate" new parameters. 
Thus, we will introduce a new set of input data, corresponding to a new situation and thus we will be able to find the answer given by the network.

Table 2. New parameters simulated with RNA

\begin{tabular}{|l|l|}
\hline \multicolumn{2}{|l|}{ INPUT } \\
\hline Layer thickness (mm) & 0,09 \\
\hline H/D Ratio & 5 \\
\hline Infill density (\%) & 20 \\
\hline OUTPUT \\
\hline Diameter (mm) & 4,0827 \\
\hline
\end{tabular}

\section{Conclusions}

The diameter of the thin shafts obtained by FDM process depends on the parameters layer thickness, $\mathrm{H} / \mathrm{D}$ ratio and infill density.

A regression equation was established after regression analysis: Diameter $=\mathrm{f}$ (parameters).

With Artificial Neural Network Toolbox in MATLAB was realized the data analyses.

The network was trained, tested and validated using the results of measurements.

After the training, the network can be used by the designer "to ask" for advice in order to "simulate" new parameters. Thus, we will introduce a new set of input data, corresponding to a new situation and thus we will be able to find the answer given by the network.

In this study, we found, for thin shafts obtained by FDM, that a large influence on the dimensional accuracy has the thickness of the printed layer, and a small influence has the filling density.

For the range of values analyzed for the $\mathrm{H} / \mathrm{D}$ ratio, the dimensional accuracy is not significantly influenced.

In the experiment we tried to obtain specimens with layer thicknesses of $0.29 \mathrm{~mm}$ and $0.39 \mathrm{~mm}$, respectively, but these were practically impossible to achieve because the cylindrical structure became unstable because it had an insufficient number of layers in the cross section of the shaft.

\section{References}

[1] Anghel, D. C., Rizea, A. D., Iordache, D. M., \& Beșliu-Gherghescu, M. L. (2019, October). Studies on the influence of design parameters on the behaviour at shock of 3D-printed components fabricated by fused deposition modelling. In IOP Conference Series: Materials Science and Engineering (Vol. 564, No. 1, p. 012014). IOP Publishing.

[2] Karamooz Ravari, M., Kadkhodaei, M., Badrossamay, M., \& Rezaei, R. (2014). Numerical Investigation on mechanical properties of cellular lattice structures fabricated by fused deposition modeling. International Journal of Mechanical Sciences, 88, 154-161.

[3] Khan ZA, Lee BH, Abdullah J. Optimization of rapid prototyping parameters for production of flexible ABS object. J Mater Process Technol 2005; 169:54-61.

[4] Kridli, G. T. (2006). Material Properties and Characterization. In Rapid Prototyping (pp. 1-22). Springer, Boston, MA.

[5] Kundera, Cz., Kozior, T. Research of the Elastic Properties of Bellows Made in SLS Technology, Advanced Materials Research, Volume: 874 pp.77-81, 2014.

[5] Mohamed O. A., Masood S. H., Bhowmik J. L., Optimization of fused deposition modeling process parameters for dimensional accuracy using I-optimality criterion, Measurement 81, 174-196.

[6] Tsouknidas, A., Pantazopoulos, M., Katsoulis, I., Fasnakis, D., Maropoulos, S., \& Michailidis, N. (2016). Impact absorption capacity of 3D-printed components fabricated by fused deposition modelling. Materials and Design, 102, 41-44. 